\title{
Neuro-ophthalmology Training in Ophthalmology Residency Programs in the United States
}

\author{
Laura L. Wayman, MD ${ }^{1}$ John J. Chen, MD, $\mathrm{PhD}^{2}$ Jacqueline A. Leavitt, MD ${ }^{3}$ \\ ${ }^{1}$ Department of Ophthalmology, Vanderbilt University, \\ Nashville, Tennessee \\ Address for correspondence Jacqueline A. Leavitt, MD, Department \\ of Ophthalmology, Mayo Clinic, 200 First Street SW, Rochester, MN
}

${ }^{2}$ Department of Neurology and Ophthalmology, Mayo Clinic, Rochester, Minnesota

${ }^{3}$ Department of Ophthalmology, Mayo Clinic, Rochester, Minnesota 55905 (e-mail: leavitt.jacqueline@mayo.edu).

Journal of Academic Ophthalmology 2018;10:e12-e15.

\begin{abstract}
Background The status of neuro-ophthalmology education in ophthalmology residency training in the United States is unknown. There are numerous articles in the literature detailing resident outcomes for surgical procedures; however, there are no articles detailing teaching of a nonprocedural specialty. There are no specific Accreditation Council for Graduate Medical Education (ACGME) requirements for neuroophthalmology training within ophthalmology residency. Each program determines the amount of neuro-ophthalmology training and level of training within ophthalmology residency. There are no publications that detail the extent of neuro-ophthalmology training during ophthalmology residency.

Objective To determine the status of neuro-ophthalmology education in ophthalmology residency training programs in the United States.

Methods A survey was sent in 2014 to residency directors and neuro-ophthalmologists of all ophthalmology residency programs who participated in the Ophthalmology Residency Matching Program in the United States to determine the amount of neuroophthalmology training that residents receive.

Results From a total of 113 ophthalmology residency programs in the United States utilizing the Ophthalmology Residency Matching Program, 104 surveys were returned ( $92 \%$ response rate). Duration of neuro-ophthalmology training ranged from 1 to 112 days, with an average of 34.5 days. Most rotations occurred within postgraduate year 2 or 3 .

Keywords

- neuro-ophthalmology

- residency

- education

Conclusion This is the first evaluation of the amount of neuro-ophthalmology training within ophthalmology residencies participating in the matching program in the United States, which demonstrates large variability among the different programs. Future studies could ascertain if there is a correlation between resident satisfaction in neuroophthalmology training and the amount of training.
\end{abstract}

The Accreditation Council for Graduate Medical Education (ACGME) currently has no time allotment requirements for particular subjects during residency training in ophthalmology. Each residency program is required to have subspecialty expertise in neuro-ophthalmology, and each resident is charged with demonstrating competence in neuro-ophthalmology. ${ }^{1}$

received

March 27, 2017

accepted after revision

December 19, 2017
DOI https://doi.org/

10.1055/s-0038-1626731. ISSN 2475-4757.
While there are strict minimum surgical requirements for the various surgical procedures, there are no predetermined requirements for the nonsurgical areas of ophthalmology; therefore, the time spent in different subspecialties greatly varies from program to program. There are several published articles that have tried to assess the satisfaction and preparation

Copyright $\odot 2018$ by Thieme Medical Publishers, Inc., 333 Seventh Avenue, New York, NY 10001, USA. Tel: +1(212) 584-4662.
License terms

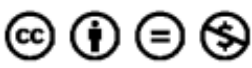


of residency training in ophthalmology either during training or shortly after graduation. ${ }^{2-4}$ Many publications have been written with regard to adequate training for procedural tasks within ophthalmology, but there are no studies that have assessed the time spent in each of the subspecialties of ophthalmology during residency training in the United States. $^{5-13}$ This includes a lack of studies assessing the time spent during ophthalmology training on the subject of neuroophthalmology, which arguably contains the most severe and life-threatening disease entities within ophthalmology.

Studies examining the level of satisfaction of ophthalmology training among residents and recent graduates have had response rates of 29.9 to $48 \% .^{2-4}$ The level of satisfaction of neuro-ophthalmology training consistently ranked in the bottom three to five subspecialties with 9 to $45 \%$ of respondents stating that they had inadequate training in neuro-ophthalmology. The Brazilian Council of Ophthalmology has mandated training units for accreditation, which delineates a minimum of $5 \%$ of training hours in neuro-ophthalmology, but $24.6 \%$ of graduates still felt deficient in neuro-ophthalmology training. ${ }^{3}$

Other survey studies performed to assess more general ophthalmology education document a continuing decrease in education and exposure to ophthalmology at the medical school level. Shah et al ${ }^{14}$ surveyed the medical student level of ophthalmology education in the United States and Canada. They received a 96\% (109) response rate from the 113 Association of University Professors of Ophthalmology (AUPO) member medical schools with associated ophthalmology department or divisions, 50\% response rate from 30 osteopathic schools, and $18 \%$ response rate from 40 allopathic medical schools that were not AUPO members. Preclinical instruction in ophthalmology ranged from 95\%(AUPO members) to 57\%(allopathic non-AUPO members). However, mandatory clinical rotations in ophthalmology occurred in only $18 \%$ of the AUPO schools, none of the osteopathic schools, and 14\% (one of seven respondents) of the allopathic schools.

Chan et $\mathrm{al}^{15}$ surveyed ophthalmology training as a component of family medicine residency programs in western Ontario. They sent an 85-question survey by mail to postgraduate year (PGY) 1 and 2 residents and had a 33.3\% response rate. Seven of the specific ophthalmic conditions that were queried, with regard to comfort managing, related to neuro-ophthalmology. The median response was "not comfortable at all" (one out of five) for optic neuritis and "somewhat comfortable" (two out of five) for the other six topics.

Ophthalmology training outside of ophthalmology residency training is very limited; therefore, we are dependent on well-trained ophthalmology residents to provide competent eye care.

Currently, the ACGME requires neuro-ophthalmology education within ophthalmology residencies, but there are no specifics about what that education should entail. In contrast, there are specific procedural requirements for residency programs (cataract: 86 , oculoplastic/orbit: 28 , laser: 24 , intravitreal injection: 10 , retina/vitreous: 10 , strabismus: 10 , cornea/ conjunctiva: 8 , keratorefractive: 6 , glaucoma: 5 , and globe trauma: 4 ) in the surgical subspecialties. This study utilized a survey to explore the amount of neuro-ophthalmology training in U.S. ophthalmology residency programs.

\section{Methods}

A 12-question survey ( - Table 1 ) was sent by email to ophthalmology residency directors (L.L.W.) and/or neuroophthalmologists (J.A.L.) of all ophthalmology residency programs participating in the Ophthalmology Residency Matching Program in the United States in 2014. A few questionnaires were distributed to neuro-ophthalmologists at a North American Neuro-ophthalmology Society (NANOS) annual meeting. Email reminders were sent, and any questions not adequately answered were queried by email. For a few unanswered emails, program directors were called on the phone, and the information was obtained from them directly or from the program assistant by J.A.L. If the program director was a neuroophthalmologist, no duplicate surveys were obtained. Time spent in neuro-ophthalmology was calibrated in days, converting a month of training into 20 days.

\section{Results}

Of the 104 (92\%) programs returning the survey, the average number of residents per program was 4.4, with a range from 2 to 9 (standard deviation [SD]: 1.76).

The average number of full-time neuro-ophthalmologist staff per program was 1.5 , with a range from 0 to 4 (SD: 0.97). Four programs responded that they did not have any neuroophthalmologists; one program sent their residents to The Johns Hopkins Hospital/Wilmer Eye Institute, one had a visiting faculty 1 day/month, one program counted the neuro-ophthalmology training within general clinic, and one had online lectures.

Eighty-one programs had a neuro-ophthalmology rotation in which the resident worked specifically with staff neuroophthalmologists. Twenty-seven programs had neuro-ophthalmology fellows, but only 20 programs utilized the fellows and the staff in training the residents, whereas in 7 programs, the residents worked only with the staff neuro-ophthalmologists.

Neuro-ophthalmology exposure to the residents was provided either as dedicated blocks of neuro-ophthalmology training or as educational opportunities interspersed throughout the year or shared with other specialties. The average number of days of neuro-ophthalmology training was 34.54 (SD: 20.63), with a range from 1 to 112 days during residency. Eight programs responded that they did not have formal neuro-ophthalmology rotations but included the neuro-ophthalmology education within shared clinics.

Most residents participated in the neuro-ophthalmology rotations during the PGY 2 (71 programs) or PGY 3 (64 programs) of training, but 18 programs had some neuroophthalmology training during PGY 4. Forty programs had neuro-ophthalmology training in multiple years of residency. In most programs, a single resident was on the neuro-ophthalmology rotation, but a few had multiple residents on their neuro-ophthalmology rotations, providing an average of 1.18 (SD: 0.47). 
Table 1 Neuro-ophthalmology training survey

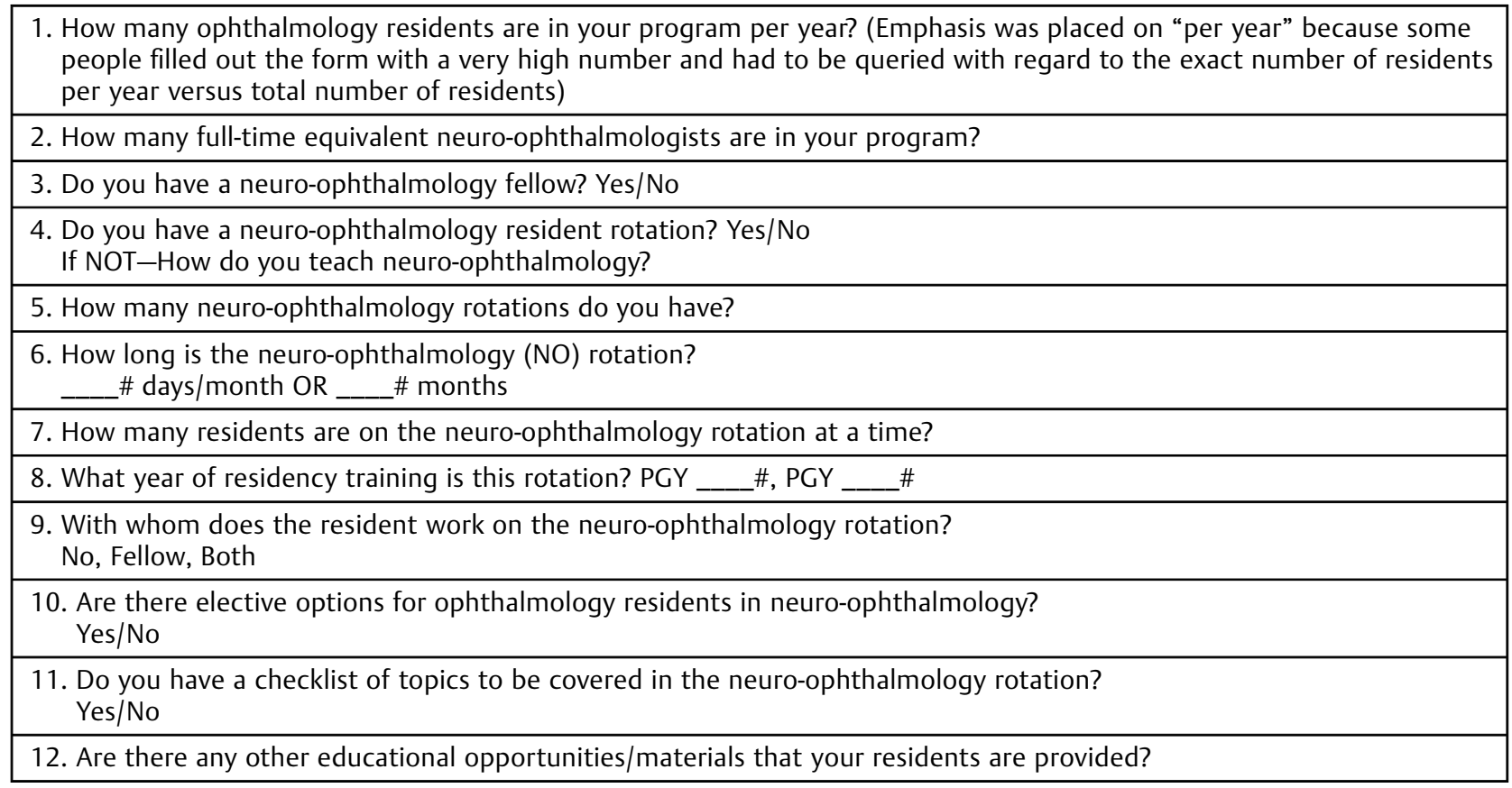

Electives for further training in neuro-ophthalmology for ophthalmology residents were available at 17 programs. Seventy programs had a checklist for neuro-ophthalmology, and a variety of other educational opportunities were available (lectures, conferences, books, journal club).

\section{Discussion}

This is the first study to investigate time involved in neuroophthalmology training within U.S. ophthalmology residencies. Currently, there are no specific ACGME time requirements for the teaching of neuro-ophthalmology within ophthalmology, unlike the detailed surgical subspecialty procedure requirements. The number of training days and residency-level neuro-ophthalmology education in U.S. ophthalmology residency programs was quite variable, ranging from 1 to 112 days, indicating that there is a large discrepancy in the neuroophthalmology exposure and teaching that U.S. ophthalmology residents receive. A recent study of Canadian ophthalmology residency training found that neuro-ophthalmology training time averaged less than 3 months ( $<60$ days using our generic calculation of 5 days/week and 4 weeks/month); this was surpassed by all other areas of training except for uveitis, pathology, oncology, low vision, and refractive surgery. ${ }^{13}$

We were able to achieve a $91 \%$ response rate for our survey; therefore, our estimates of residency training in neuro-ophthalmology are expected to be quite accurate. Recent survey response rates in ophthalmology have ranged from 12.5 to $77 \%{ }^{5,16}$ thus, our study has the highest response rate to date.

One limitation of the study is that some programs did not have a discreet neuro-ophthalmology block. Although a small caveat, calculation of the number of training days in programs without a dedicated neuro-ophthalmology block was more difficult to ascertain when the neuro-ophthalmol- ogy training occurred in general clinics or clinics shared with oculoplastics/orbit. The best estimate, in terms of days of training, was made by each of the program directors or neuro-ophthalmologists.

Using a survey with a $91 \%$ response rate, we were able to provide an accurate estimate of neuro-ophthalmology exposure during ophthalmology residency training in the United States, highlighting a very large variability among different residency training programs. Further studies will be needed to assess what factors of neuro-ophthalmology training might correlate with residents' and graduates' confidence in neuroophthalmology and Ophthalmic Knowledge Assessment Program (OKAP) and/or board scores in neuro-ophthalmology.

\section{Conclusion}

This is the first evaluation of the amount of neuro-ophthalmology training that occurs within ophthalmology residencies participating in the matching program in the United States, which demonstrates large variability in neuro-ophthalmology training among the different programs. Future studies could evaluate for a correlation between residents' satisfaction in neuro-ophthalmology training and the amount of training.

\section{Presentations}

This article was presented at the North American NeuroOphthalmology Society Meeting in 2016.

\section{Funding}

This work was supported in part by an unrestricted grant from Research to Prevent Blindness, New York.

\section{Conflict of Interest}

None declared. 


\section{References}

1 Accreditation Council for Graduate Medical Education - Common Program Requirements. Available at: http://www.acgme.org/Portals/0/PFAssets/ProgramRequirements/CPRs_07012016.pdf. Accessed December 15, 2017

2 Zhou AW, Noble J, Lam WC. Canadian ophthalmology residency training: an evaluation of resident satisfaction and comparison with international standards. Can J Ophthalmol 2009;44(05):540-547

3 Millán T, de Carvalho KM. Satisfaction with ophthalmology residency training from the perspective of recent graduates: a cross-sectional study. BMC Med Educ 2013;13:75

4 McDonnell PJ, Kirwan TJ, Brinton GS, et al. Perceptions of recent ophthalmology residency graduates regarding preparation for practice. Ophthalmology 2007;114(02):387-391

5 Yeu E, Reeves SW, Wang L, Randleman JB; ASCRS Young Physicians and Residents Clinical Committee. Resident surgical experience with lens and corneal refractive surgery: survey of the ASCRS Young Physicians and Residents Membership. J Cataract Refract Surg 2013;39(02):279-284

6 Golden RP, Krishna R, DeBry PW. Resident glaucoma surgical training in United States residency programs. J Glaucoma 2005;14 (03):219-223

7 Shah VA, Reddy AK, Bonham AJ, Sabates NR, Lee AG. Resident surgical practice patterns for vitreoretinal surgery in ophthalmic training programs in the United States. Ophthalmology 2009;116 (04):783-789
8 Rowden A, Krishna R. Resident cataract surgical training in United States residency programs. J Cataract Refract Surg 2002;28(12): 2202-2205

9 Kwon RO, Shah VA, Krishna R, Hausheer J. Resident laser in situ keratomileusis surgical training in United States residency programs. J Cataract Refract Surg 2009;35(09):1629-1632

10 Wong RK, Ventura CV, Espiritu MJ, et al. Training fellows for retinopathy of prematurity care: a Web-based survey. J AAPOS 2012;16(02):177-181

11 Binenbaum G, Volpe NJ. Ophthalmology resident surgical competency: a national survey. Ophthalmology 2006;113(07):1237-1244

12 Oetting TA. Surgical competency in residents. Curr Opin Ophthalmol 2009;20(01):56-60

13 Le K, Bursztyn L, Rootman D, Harissi-Dagher M. National survey of Canadian ophthalmology residency education. Can J Ophthalmol 2016;51(03):219-225

14 Shah M, Knoch D, Waxman E. The state of ophthalmology medical student education in the United States and Canada, 2012 through 2013. Ophthalmology 2014;121(06):1160-1163

15 Chan TY, Rai AS, Lee E, Glicksman JT, Hutnik CM. Needs assessment of ophthalmology education for primary care physicians in training: comparison with the International Council of Ophthalmology recommendations. Clin Ophthalmol 2011;5:311-319

16 Cruz OA, Pole CJ, Thomas SM. Burnout in chairs of academic departments of ophthalmology. Ophthalmology 2007;114(12): $2350-2355$ 